\title{
The description-experience gap in risky choice in nonhuman primates
}

\author{
Sarah R. Heilbronner ${ }^{1}$ - Benjamin Y. Hayden ${ }^{2}$
}

Published online: 19 August 2015

(C) Psychonomic Society, Inc. 2015

\begin{abstract}
Risk attitudes in humans depend on the format used to present the gamble: we are more risk-averse for common gambles in the gains domain whose properties are described to us verbally than for those whose properties we learned about solely through experience. This difference, which constitutes part of the description-experience gap, is important, because it highlights the role of knowledge acquisition in decision-making. The reasons for the gap remain obscure, but could depend upon uniquely human cognitive abilities, such as those associated with language. Thus, the gap may or may not extend to nonhuman animals. For this study, rhesus monkeys performed a novel task in which the properties of some gambles were explicitly cued (described), whereas others were learned through previous choices (experienced). Our monkeys displayed a description-experience gap. Overall, monkeys were more risk-seeking for experienced than for described gambles. This difference was observed for a range of gamble probabilities (from $20 \%$ to $80 \%$ likelihood of payoff), indicating that it is not limited to low probability events. These results suggest that the descriptionexperience gap does not depend on uniquely human cognitive abilities, such as those associated with language, and support the idea that epistemic influences on risk attitudes are evolutionarily ancient.
\end{abstract}

Sarah R. Heilbronner

sarah.heilbronner@gmail.com

1 Department of Pharmacology and Physiology, University of Rochester School of Medicine and Dentistry, 601 Elmwood Ave, Box 711, Rochester, NY 14642, USA

2 Department of Brain and Cognitive Sciences and Center for Visual Sciences, University of Rochester, Rochester, NY 14627, USA
Keywords Animal cognition · Animal learning · Decision making . Choice behavior

\section{Introduction}

Risk sensitivity guides our decisions in simple and complex situations as diverse as foraging, mating, and paragliding. When choosing amongst gambles that offer rewards (the gains domain), humans are typically risk-averse - meaning that we prefer a known, safe option to a risky one, even when the risky one may have a slightly higher expected value (EV) (Bernoulli, 1738; Kahneman \& Tversky, 1979). Risk is operationalized as the uncertainty in the possible outcomes of a decision (Sharpe, 1964; Heilbronner and Hayden, 2013).

This robust risk aversion is commonly observed in gambles where probabilities and rewards are described to the subject, for example, on a questionnaire. However, preferences change dramatically when the properties of the gamble are learned from experience, rather than described - a phenomenon known as the description-experience gap (Camilleri \& Newell 2013; Hau, Pleskac, Kiefer, \& Hertwig, 2008; Hertwig, Barron, Weber, \& Erev, 2004; Hertwig \& Erev, 2009; Ludvig \& Spetch, 2011).

The description-experience gap often is thought to arise from an underweighting of experienced low probabilities (Hertwig et al., 2004; Ungemach, Chater, \& Stewart, 2009) versus an overweighting of described low probabilities $(<20 \%$ occurrence) (Kahneman \& Tversky, 1979). However, even with gambles of equally probable outcomes $(50 \% / 50 \%)$, risk-seeking for gains is enhanced for experienced gambles relative to described ones (Ludvig \& Spetch, 2011). Moreover, these preferences persist even after extensive training with the experienced option, suggesting that limited exposure to experienced options is not driving the gap. Thus, it seems 
we are generally more risk-seeking for common, experienced gambles than for described ones.

The reasons for the description-experience gap in risky choice remain unclear. One possibility is that there is something special about language, or about cultural learning, that motivates the use of different strategies for verbally described gambles. We therefore wondered whether the same gap would be observed in nonhuman animals. Prior studies have found that, while general risk preferences may vary considerably across species (Bateson \& Kacelnik, 1996; De Petrillo, Ventricelli, Ponsi, \& Addessi, 2015; Heilbronner, Hayden, \& Platt, 2009; Heilbronner, Rosati, Stevens, Hare, \& Hauser, 2008), many of the fundamental decision-making biases found in humans can be replicated in nonhuman animals. For example, nonhuman primates, like humans, are ambiguity-averse (Hayden, Heilbronner, \& Platt, 2010; Rosati \& Hare, 2011) and are susceptible to loss versus gain framing effects (Chen, Lakshminarayanan, \& Santos, 2006; Krupenye et al., 2015). If the description-experience gap is shared with nonhuman animals, it is unlikely to depend upon uniquely human cognitive capacities. While such decisionmaking biases could conceivably evolve independently in two or more species, by testing a nonhuman animal that is closely related to humans - the rhesus macaque - we hope to identify a possible nonhuman homology of the description-experience gap.

Of course, nonhuman animals do not have language, so ostensibly the only choices that can be offered are those from experience. However, the description-experience dichotomy can be understood as a continuum of uncertainty, rather than two binary states (Camilleri \& Newell 2013; Hertwig \& Erev, 2009). Accordingly, it is possible to compare options that are relatively more described with those that are relatively more experienced. We previously developed a task in which probabilities of winning and losing a gamble were explicitly cued to monkeys on a trial-to-trial basis (Hayden, Heilbronner, Pearson, \& Platt, 2011; Hayden et al., 2010). Monkeys chose between rectangles divided into portions reflecting the probabilities of various rewards. They successfully chose gambles with higher probabilities of wins, even for unique bar combinations (Hayden et al., 2010). Thus, monkeys treated these stimuli as if they were explicit probability cues that could change from trial to trial, much like humans treat described options. Furthermore, when probabilities are explicitly cued, monkeys overweight low probabilities, much as humans do with described gambles (cf. Stauffer, Lak, Bossaerts, \& Schultz, 2015). Thus, explicit probability cues seem to be a viable implementation of described gambles for macaques. However, risk attitudes with experienced and described gambles have never been directly compared in macaques (or any other nonhuman animal). We perform this comparison here, using a new Description vs. Experience Gambling Task.
We found that our rhesus macaque subjects were more riskseeking for experienced common gambles than for described ones (20-80\% win probabilities). Furthermore, when directly comparing experienced and described gambles, the monkeys showed a bias for experienced ones. These results have several implications for the field. First, the description-experience gap extends to a nonhuman primate species, and thus is unlikely to depend on uniquely human cognitive processes. Second, our results establish the generality of the descriptionexperience gap across many probabilities. Third, by collecting thousands of trials, we were able to reduce the likelihood of biased sampling. Finally, because these monkeys were riskseeking for gains, we were able to ascertain that the gap does not depend on risk aversion for described gambles. All of these represent important advances in understanding the evolutionary and cognitive origins of risky choice.

\section{Methods}

Three adult male rhesus macaques (Macaca mulatta; subjects B, J, K) performed a computerized Description vs. Experience Gambling Task. On each trial, monkeys chose between two options, a described and an experienced gamble ( $80 \%$ of trials), a described gamble and a safe option ( $10 \%$ of trials), or an experienced gamble and a safe option ( $10 \%$ of trials). The task was written in Matlab using Psychtoolbox and Eyelink Toolbox. Eye positions were sampled by a camera system (SR Research, Osgoode, Ontario, Canada). A small mount affixed to the head maintained head position during performance. A solenoid valve controlled the delivery duration of fluid rewards (water).

On each trial, a fixation dot appeared in the center of a black computer screen placed three feet in front of the monkey. Monkeys initiated the trial by fixating on this central dot. Fixation for $500 \mathrm{~ms}$ produced the appearance of the two offers along with the disappearance of the dot. The two offers appeared on the monitor, simultaneously, to the left and right of the fixation dot location. Monkeys chose the preferred option by shifting gaze to it and maintaining that gaze for $200 \mathrm{~ms}$. Following the hold period, the gambles were resolved and the reward given. Then, a 1.5-second inter-trial interval began.

Experienced gambles were one of five emotionally neutral nature scenes (Fig. 1). Each scene corresponded to a win probability of $20 \%, 35 \%, 50 \%, 65 \%$, or $80 \%$. Each scene was associated with a specific win probability, and the mapping between specific monkey and specific win probability was different for each of the three monkeys. Scenes were chosen randomly with equal likelihood. Described gambles were vertically oriented rectangles, $300 \times 80$ pixels, divided into a red and a blue portion; the relative sizes of these portions indicated the probability of losing $(0 \mu \mathrm{L}$ of water) and winning the large reward $(250 \mu \mathrm{L}$ of water $)$, respectively 


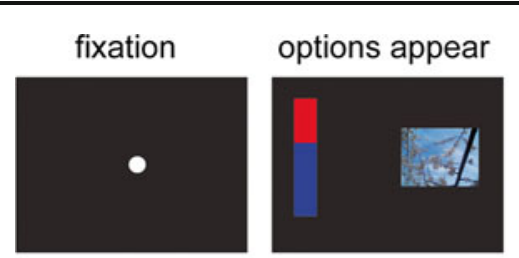

$0.5 \mathrm{sec}$

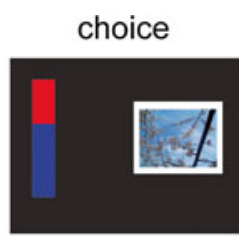

$0.2 \mathrm{sec}$ reward delivery

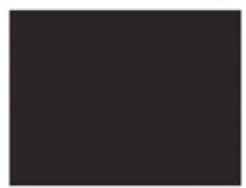

0 to $0.25 \mathrm{sec}$

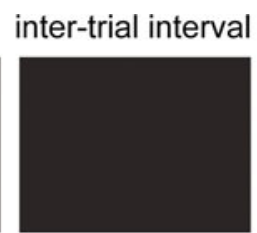

$1.5 \mathrm{sec}$
Described gambles

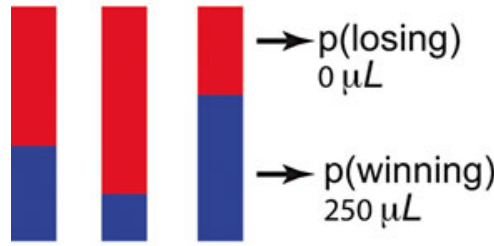

Experienced gambles

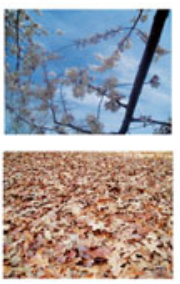

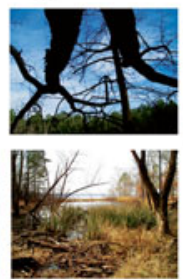

Safe option

$(125 \mu L)$
Fig. 1 Task design. Each trial begins when the monkey fixates on a central point on a computer monitor. Following $0.5 \mathrm{sec}$ of holding fixation, the options appear. These options could be every combination of described, experienced, and safe, although both options were not allowed to be drawn from the same category. The monkey makes a choice by shifting his gaze to one of the targets, then holding it there for $0.2 \mathrm{sec}$. A white rectangle outlines the chosen option, and then the reward is delivered. A 1.5 -sec inter-trial interval follows
(Fig. 1). Described risky options were chosen from a uniform distribution of probabilities from 0 to $100 \%$, using $1 \%$ increments. The safe option was indicated by a gray bar of the same size, and, when chosen, always delivered $125 \mu \mathrm{L}$ of water. Thus, the expected value (i.e., EV, or probability of winning * size of reward following win) of the safe option was equal to the EV of a $50 \%$ gamble, which also was equal to the mean of all gambles.

All subjects had extensive prior experience with the described gamble options. The experienced gamble options were newly learned for this study. Subjects B and J had $\sim 10,000$ trials of experience and subject $\mathrm{K}$ had $\sim 5000$ trials of experience, more than 2 weeks of daily sessions conducted Monday through Friday, before the data described were collected. This training period served to mitigate possible biases due to novelty-seeking or complexity.

Note that our task constitutes a partial-feedback paradigm, in which each choice produces an outcome, but no information is revealed about the unchosen option (Hertwig \& Erev, 2009). Both partial feedback and full feedback paradigms have replicated the description-experience gap in humans (Barron \& Erev, 2003; Yechiam \& Busemeyer, 2006). Analyses were performed using custom scripts in Matlab. Alpha levels were set at 0.05 , and all comparisons are two-tailed.

\section{Results}

\section{Monkeys understood the basic structure of the task}

We collected preference data in the Description vs. Experience Gambling Task in three monkeys (Fig. 1 for task schematic). We collected 10,000 trials in monkey B, 10,000 trials in monkey J, and 5,000 trials in monkey K. Each trial consisted of a choice between an experienced and a described gamble or an experienced or described gamble and a safe option. Experienced gambles consisted of one of five photographic images (Fig. 1) and had a win probability of $20 \%, 35 \%, 50 \%, 65 \%$, or $80 \%$. Described gambles consisted of a two-color rectangle divided into a red and a blue portion; the relative size of the two portions indicated the probabilities of losing and winning, respectively.

To confirm that the monkeys understood the structure of the task, we first established that they preferred gambles with higher win probabilities (Fig. 2). For experienced gambles, all three monkeys preferred higher probability options (and thus greater EVs; Fig. 2a; regression of preference against EV; subject B: $r=0.410, \mathrm{n}$ (trials) $=10,000,95 \%$ CI [0.394, $0.426] ; \mathrm{J}: \mathrm{r}=0.537, \mathrm{n}=10,000,95 \%$ CI [0.523, 0.55]; K: $\mathrm{r}=$ $0.187, \mathrm{n}=5,000,95 \%$ CI $[0.161,0.213] ; P<0.0001$ in all cases). Likewise, for described gambles, all three monkeys preferred options with higher probability (Fig. 2b; B: $\mathrm{r}=$ $0.481, \mathrm{n}=10,000,95 \%$ CI [0.466, 0.495]; J: $\mathrm{r}=0.578, \mathrm{n}=$ $10,000,95 \% \mathrm{CI}[0.565,0.59] ; \mathrm{K}: \mathrm{r}=0.539, \mathrm{n}=5,000,95 \% \mathrm{CI}$ [0.52, 0.558]; all are significant, $P<0.0001)$.

\section{Monkeys were risk-seeking, but more so for experienced than for described gambles}

We next assessed the monkeys' preference for risk in this task. All three monkeys preferred the experienced risky option to the safe option (Fig. 3a). When choosing between an experienced gamble (of all win probabilities) and a safe option (note that the mean EVs of both types are identical), subject B chose risky on $86.94 \%$ of trials $(\mathrm{n}=1095$ trials), 95\% CI [84.80, 88.88]; J chose risky on $71.03 \%$ of trials $(n=1039$ trials $), 95 \%$ 
A

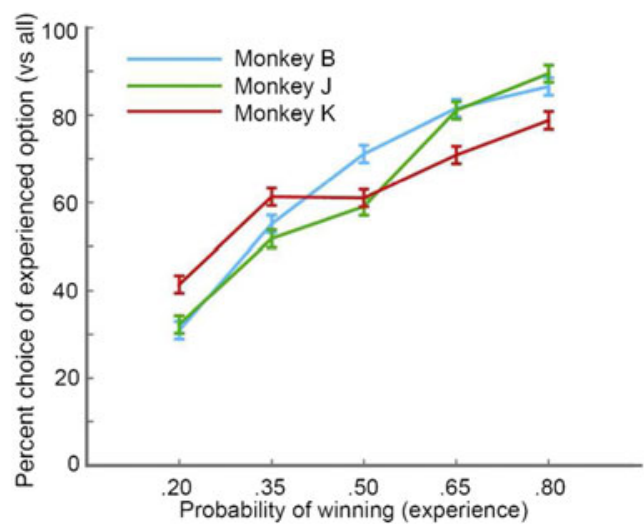

Fig. 2 Choice of risky options increased as their win probabilities (and thus EVs) increased. a Experience condition. b Description condition (options are binned in $10 \%$ increments, with the $\mathrm{x}$-axis label showing

CI [68.17, 73.77]; $\mathrm{K}$ chose risky on $81.52 \%$ of trials $(\mathrm{n}=525$ trials), 95\% CI [77.93, 84.75]. These are all significant with a binomial test vs. $50 \%, P<0.00001$. This replicates earlier findings showing that monkeys are risk-seeking for experienced gambles (Hayden, Nair, et al., 2008; Heilbronner et al., 2011; McCoy \& Platt, 2005; Xu \& Kralik, 2014).

Similarly, all three monkeys preferred the described risky option to the safe option (Fig. 3a). Overall, B chose the risky option on $63.48 \%$ of trials $(\mathrm{n}=879$ trials), $95 \%$ CI [60.20, 66.67]; J chose risky on $59.01 \%$ of trials ( $\mathrm{n}=966$ trials), $95 \%$ CI [55.83, 62.13]; K chose risky on $63.80 \%$ of trials $(n=489$ trials), 95\% CI [59.37, 68.07]; all three biases are significantly greater than indifference $(P<0.0001$, binomial tests). This replicates earlier reports showing that monkeys are risk-

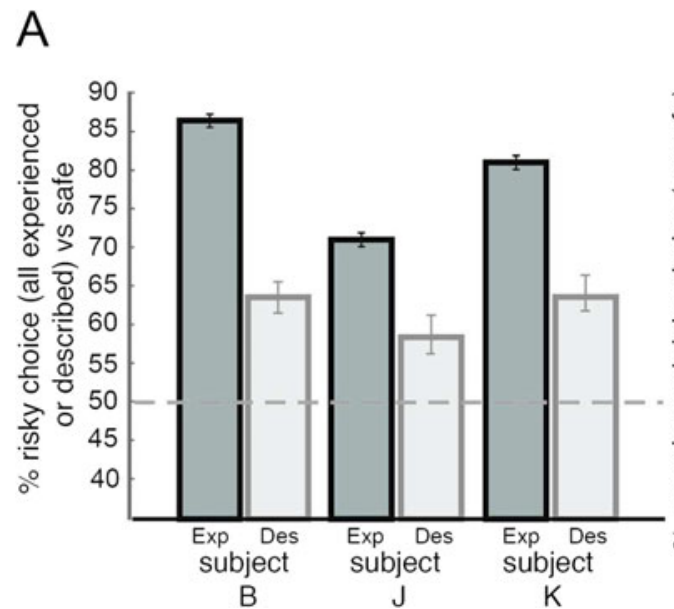

Fig. 3 Monkeys were risk-seeking for both experienced and described gambles, but were more risk-seeking when confronted with experienced options than when confronted with described options. a Percent choice of risky option versus safe option only, separated by experienced risky options (dark gray, all win probabilities included) and described risky options (light gray, all win probabilities included). All bars are $>50 \%$, indicating risk-seeking. Percent choices of experienced options (dark gray)
B

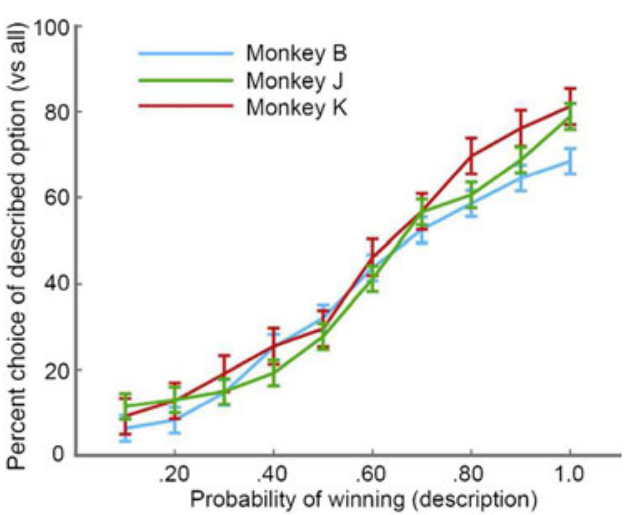

the upper bound of each bin). Data shown are experienced and described options versus all other options (risky experienced, risky described, and safe, for all win probability increments), \pm SEM

seeking for described gambles (Hayden et al., 2010; Seo \& Lee, 2009; So \& Stuphorn, 2010; Stauffer, Lak, Bossaerts, \& Schultz, 2015; Strait et al., 2014; Strait et al., 2015).

Notably, the monkeys' bias towards risk-seeking was stronger for experienced gambles than for described ones (Figs. 3a-b) (independent-samples $t$ tests performed on choices in experience vs. description conditions-B: $\mathrm{t}(1972)$ $=12.70, \mathrm{~d}=0.57, P<0.00001 ; \mathrm{J}: \mathrm{t}(2003)=5.69, \mathrm{~d}=0.25$, $P<0.00001 ; \mathrm{K}: \mathrm{t}(1012)=6.47, \mathrm{~d}=0.41, P<0.0001)$. Combining the data for all three monkeys (and binning $\pm 10 \%$ for the described gambles), a significant increase in riskpreference for experienced as opposed to described gambles was observed at each probability level $(P<0.01$ in each of the five probabilities).

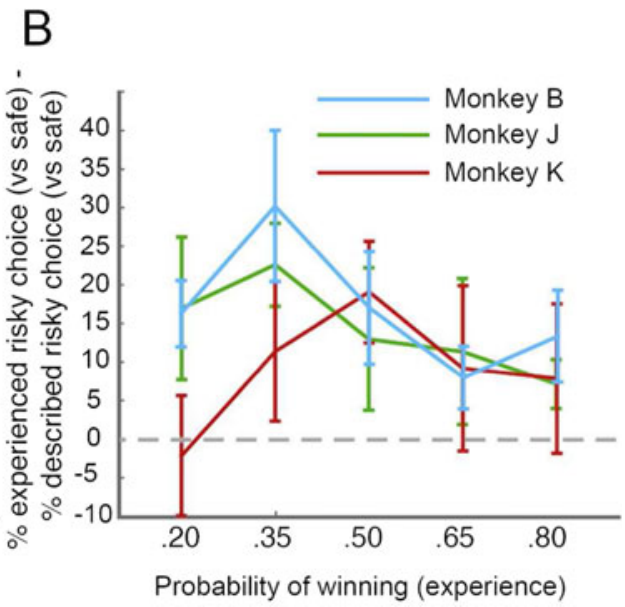

are higher than percent choices of described options (light gray), indicating that subjects were more risk-seeking for experienced than described risky options. b Plot of difference in proportion of choices of the risky (as opposed to safe) option for experienced and described gambles. Values greater than zero indicate greater risk-seeking for experienced versus described gambles with matched expected values. Data for described gambles are binned at $\pm 10 \%$ of the experienced win probability 


\section{Monkeys preferred experienced gambles to described ones with equivalent probabilities}

We next examined preferences for described and experienced gambles directly by looking at trials that pitted them against each other ( $80 \%$ of all trials). All three monkeys reliably preferred experienced to described gambles (Fig. 4). Indeed, when facing a $50 \%$ experienced gamble, monkeys chose it over the described gamble more than half the time (B: $71.12 \%, \mathrm{n}=2029$ trials, $95 \%$ CI $[69.09,73.08]$; J: $59.07 \%$, $\mathrm{n}=1962$ trials, $95 \%$ CI $[56.86,61.26] ; \mathrm{K}: 57.03 \%, \mathrm{n}=1015$ trials, $95 \%$ CI [53.93, 60.11]; $P<0.0001$ in all cases, binomial test). Considering the entire set of gambles (that is, including the $20 \%, 35 \%, 50 \%, 65 \%$, and $80 \%$ gambles), monkeys chose the experienced over the described more than half the time as well (B: $65.01 \%, \mathrm{n}=8026$ trials, 95\% CI [63.96, 66.06]; J: $62.58 \%, \mathrm{n}=7995$ trials, $95 \% \mathrm{CI}[61.50,63.64]$; K: $58.06 \%, \mathrm{n}$ $=3986$ trials, $95 \% \mathrm{CI}[56.50,59.59] ; P<0.0001$ in all cases, binomial test). For both of these analyses, the described and experienced options were stochastically equal in EV, so neutrality would imply no bias and thus a 50\% preference. Indeed, a significant preference for experienced over described gambles was observed for each subject at each probability $(P<$ 0.01 in each case, binomial test; Fig. $4 \mathrm{~b})$, with the exception of subject $\mathrm{K}$ at the value of $80 \%(P=0.58)$.

We also observed a significant interaction between probability and gamble type. Combining the three monkeys and binning the win probabilities of the described options to match the experienced, preference for experienced options over the described one was greatest when the odds of winning were lowest (Fig. 4b). To measure this effect, we correlated the description-experience gap with probability ( $\mathrm{r}$ $=-0.78,95 \%$ CI $[-0.0 .92-0.45], P<0.001)$. One possible explanation for this interaction is a ceiling

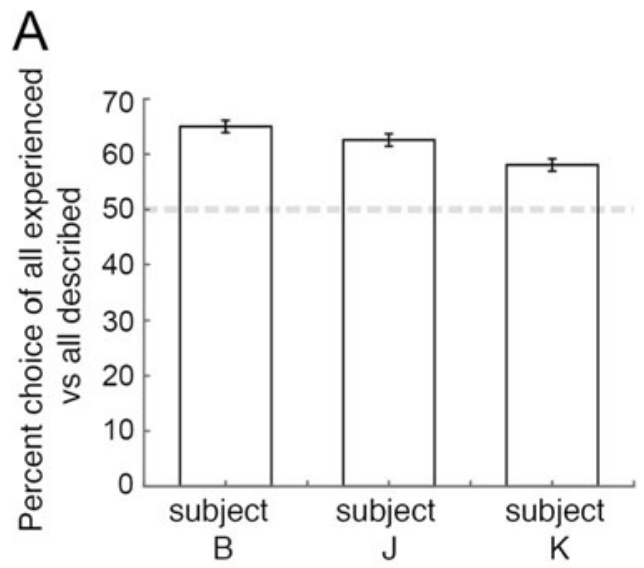

Fig. 4 Monkeys preferred options learned from experience to described options. a Percent choosing experience option (versus description option) when these gambles are directly pitted against each other on the same trial, averaged across all win probabilities. Gray dashed line indicates description-experience neutrality $(50 \%)$. b Percent choosing experience effect in risk-seeking (Fig. 4), and thus the importance of this effect should not be overstated.

\section{Discussion}

Humans' risk preferences change depending upon whether gambles are presented using symbolic presentations or whether choices are based solely on learned associations (Camilleri \& Newell, 2013; Hau et al., 2008; Hertwig et al., 2004; Hertwig \& Erev, 2009; Ludvig \& Spetch, 2011). For gambles with $50 \% / 50 \%$ probabilities, for example, humans are more risk-seeking for experienced than described ones (Ludvig \& Spetch, 2011). We show that the description-experience gap occurs in rhesus monkeys as well. Rhesus monkeys, whose ancestors diverged from humans' only $\sim 25$ million years ago, represent an important nonhuman animal species with which to test the idea that the existence of the gap predates the evolution of human-specific cognitive abilities. Although we cannot rule out the possibility of convergent evolution, this finding does suggest that the description-experience gap is not exclusively a by-product of humans' specialized symbolic representation systems. Likewise, this finding suggests that the description-experience gap is not a consequence of our evolved capacity for language or our particular cultural training. Instead it supports the hypothesis that the descriptionexperience gap reflects a fundamental similarity in the way our shared cognitive systems handle the difference between inferences based on explicit cues and trial-and-error learning.

One limitation of our study is that the described condition is not strictly identical to the described condition usually used in human studies (but see Jessup et al., 2008 and Fitzgerald et al., 2010 for methodologically similar human studies). In particular, monkeys don't have language, so we had to teach

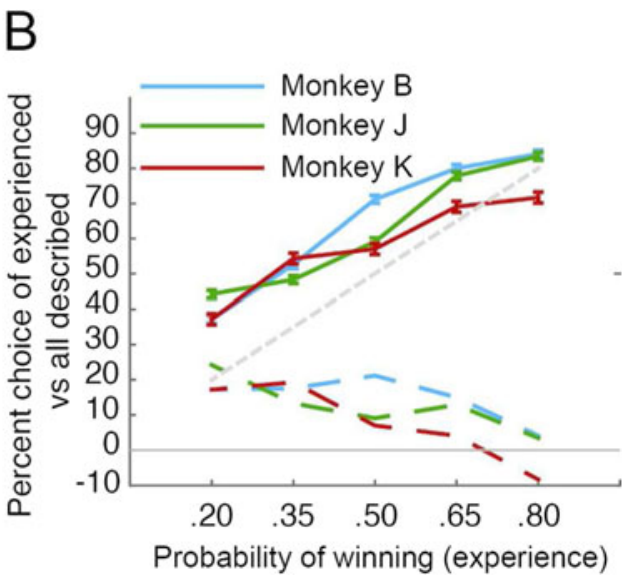

option, according to probability of winning, versus all described options, when these are directly pitted against each other. Dashed colored lines indicate results from each subject minus description-experience neutrality (gray dashed line), and thus represent the description-experience gap 
them a novel symbolic system that is used exclusively for gambles. In contrast, humans use numbers and language throughout our daily lives. Moreover, for our monkeys, all outcomes were experienced and trials were structured serially, whereas in human studies, described gambles are normally hypothetical, and there is often no opportunity for learning between trial types. These changes were made out of necessity, and, in our view, make the described gambles in our task something of an intermediate case between canonical experienced and described gambles. Thus, we propose that experience and description lie on a continuum and that in our paradigm the two gamble types are more similar to each other than they are in human studies. In any case, we believe enough core features surrounding the certainty associated with each option's outcomes are preserved that one can fruitfully-if tentatively-compare these results with those obtained in humans.

The psychological bases of the description-experience gap in risky choice are unclear. One possible explanation is that the description-experience gap is a statistical artifact of decision-makers' sampling bias rather than a true reflection of preferences (Fox \& Hadar, 2006). Indeed, increasing the number of exposures seems to reduce the description-experience gap, although does not eliminate it (Hau et al., 2008; Ungemach et al., 2009). Our own data contain thousands of trials of exposure, so this sampling bias is unlikely to apply. Perhaps the most common explanation for the gap, however, is that experiential learning involves an underweighting of rare events, versus an overweighting of rare, described events (Hertwig et al., 2004; Ungemach et al., 2009; Kahneman \& Tversky, 1979). Unfortunately, this explanation does not apply to the recently demonstrated description-experience gap for common (50/50), rather than rare, gambles, suggesting that other factors may be involved (Ludvig \& Spetch, 2011). Our results confirm this recent finding, and extend it, both to a nonhuman animal species, and to a broader range of probabilities $(20 \%-80 \%)$.

One possibility is that experienced options benefit from an information bonus: subjects choose them because they offer both a reward and potentially useful information about reward outcomes and probabilities (Blanchard, Hayden, \& Bromberg-Martin, 2015; Bromberg-Martin \& Hikosaka, 2009; Niv \& Chan, 2011). Although the long training period we used meant that the information that could be gleaned from the experienced option was greatly reduced in our study, monkeys may still value information in stable environments after thousands of trials of experience (Blanchard et al., 2015; Bromberg-Martin \& Hikosaka, 2009). This residual, perhaps even instinctive, uncertainty about an experienced option's outcomes may motivate risky choices (Hayden, Heilbronner, Nair, \& Platt, 2008; Heilbronner \& Hayden, 2013). This motivation may reflect an inherent belief that the environment is more unstable than it actually is. We also cannot completely rule out the possibility of a novelty or stimulus complexity bonus for experienced options, or even a training effect (described options were learned long before experienced ones), which may interact with these results (Wittmann et al., 2008; Pearson et al., 2009).

One unique aspect of our study is that rhesus monkeys, unlike humans, are reliably risk-seeking for rewards (Heilbronner \& Hayden, 2013; Xu \& Kralik, 2014). Thus, we show that even though monkeys are risk-seeking for described gambles, they become even more risk-seeking for experienced ones. In other words, the description-experience gap is robust enough that it is preserved in contexts where risk attitudes are flipped around the axis of neutrality. These data therefore suggest that experience produces a consistent bias towards risk-seeking, rather than a regression towards neutrality. Thus, just as species differences affect risk preferences, so too can biases (such as framing effects or ambiguity aversion).

The present results are intriguing in light of patterns of risk and ambiguity preferences. As it is normally defined, the term risk refers to choices in which all details of the gamble, aside from its outcome, are known to the decision-maker. In contrast, ambiguity refers to additional uncertainty related to the probabilities associated with outcomes (Ellsberg, 1961). For example, a roulette wheel offers a risky gamble but an unseen roulette wheel with an unknown ratio of red to black numbers is both risky and ambiguous. Humans prefer risky options to ambiguous ones (Curley, Yates, \& Abrams, 1986; Einhorn \& Hogarth, 1985; Ellsberg, 1961), as do monkeys (Hayden et al., 2010). These results suggest that certainty about probabilities at the time of choice is prized. In our task, one might expect therefore that experienced options are more ambiguous, and would thus be avoided. However, monkeys' preferences for experienced rather than described options would suggest the opposite. This simultaneous ambiguity aversion and preference for experienced gambles is a puzzle both for human and monkey results. One speculative possibility is that both species are information-seeking but selectively prefer information of moderate complexity (neither too complex nor too simple) to maximize learning (Kidd, Piantadosi, \& Aslin, 2012).

The present results highlight the often-ignored role of context and presentation in determining risk attitudes. For example, salient memories of prior winning gambles, whether recalled naturally or primed, can induce greater risk-taking (Hayden, Heilbronner, et al., 2008; Ludvig, Madan, Pisklak, \& Spetch, 2014; Ludvig, Madan, \& Spetch, 2015). More broadly, these results endorse the idea that risk attitudes in nonhuman animals are strongly and predictably mediated by psychological factors - just as they are in humans (Tversky \& Kahneman, 1981). These psychological factors include local reward context, delays between trials, anchoring effects, and social context, in addition to the presentation biases described here (Hayden, Heilbronner, et al., 2008; Madan, Ludvig, \& 
Spetch, 2014; Rachlin, Raineri, \& Cross, 1991; Silberberg, Murray, Christensen, \& Asano, 1988). Interestingly, the use of symbolic cues to indicate task parameters, rather than learning these from experience, alters performance in discounting tasks as well (Pearson et al., 2010; Blanchard et al., 2013).

As others have pointed out, the disconnect in preferences between described and experienced gambles has far-reaching implications for both individual decision-making as well as economic policy (Camilleri \& Newell 2013; Hertwig \& Erev, 2009). Our results support this conclusion, but also offer support for the notion of a continuum of experience versus description. Probabilities may not need to be verbally described in order to be (nearly) explicitly cued. A broader understanding of how probabilities are represented across species will help us develop generalizable models of real-world decision-making.

Acknowledgments The authors thank Meghan Castagno, Marc Mancarella, Giuliana Loconte, and Julian Nin for help with data collection. This work was supported by grants to BYH from the Templeton Foundation and the Alfred P. Sloan Foundation.

\section{References}

Barron, G., \& Erev, I. (2003). Small feedback-based decisions and their limited correspondence to description-based decisions. Journal of Behavioral Decision Making, 16, 215-233.

Bateson, M., \& Kacelnik, A. (1996). Rate currencies and the foraging starling: the fallacy of the averages revisited. Behavioral Ecology, 7(3), 341-352.

Bernoulli, D. (1738). Specimen theoriae novae de mensura sortis (Exposition of a new theory on the measurement of risk). Comentarii Acad. Scient. Petropolis (translated in Econometrica), 5(22), 23-36.

Blanchard, T. C., Pearson, J. M., \& Hayden, B. Y. (2013). Postreward delays and systematic biases in measures of animal temporal discounting. Proceedings of the National Academy of Science, 110(38), 15491-15496.

Blanchard, T. C., Hayden, B. Y., \& Bromberg-Martin, E. S. (2015). Orbitofrontal cortex uses distinct codes for different choice attributes in decisions motivated by curiosity. Neuron, 85(3), 602-614.

Bromberg-Martin, E. S., \& Hikosaka, O. (2009). Midbrain dopamine neurons signal preference for advance information about upcoming rewards. Neuron, 63(1), 119-126.

Camilleri, R., \& Newell, B R. (2013). Mind the gap? Description, experience, and the continuum of uncertainty in risky choice. In V. S. Chandrasekhar Pammi \& N. Srinivasan (Eds.), Progress in Brain Research (Vol. 202): Elsevier, Amsterdam: The Netherlands.

Chen, M. K., Lakshminarayanan, V., \& Santos, L. (2006). How Basic Are Behavioral Biases? Evidence from Capuchin Monkey Trading Behavior. Journal of Political Economy, 114(3), 517-537.

Curley, S. P., Yates, J. F., \& Abrams, R. A. (1986). Psychological sources of ambiguity avoidance. Organizational Behavior and Human Decision Processes, 38(2), 230-256.

De Petrillo, F., Ventricelli, M., Ponsi, G., \& Addessi, E. (2015). Do tufted capuchin monkeys play the odds? Flexible risk preferences in Sapajus spp. Animal Cognition, 18, 119-130.

Einhorn, H. J., \& Hogarth, R. M. (1985). Ambiguity and uncertainty in probabilistic inference. Psychological Review, 92, 433-461.
Ellsberg, D. (1961). Risk, Ambiguity, and the Savage Axioms. The Quarterly Journal of Economics, 75(4), 643-669.

FitzGerald, T. H. B., Seymour, B., Bach, D. R., \& Dolan, R. J. (2010). Differentiable neural substrates for learned and described value and risk. Current Biology, 20(20), 1823-1829.

Fox, C. R., \& Hadar, L. (2006). "Decisions from experience" = sampling error + prospect theory: Reconsidering Hertwig, Barron, Weber \& Erev (2004). Judgment and Decision Making, 1(2), 159-161.

Hau, R., Pleskac, T. J., Kiefer, J., \& Hertwig, R. (2008). The experiencedescription gap in risky choice: The role of sample size and experienced probabilities. Journal of Behavioral Decision Making, 21(5), 493-518.

Hayden, B. Y., Heilbronner, S. R., Pearson, J. M., \& Platt, M. L. (2011). Surprise signals in anterior cingulate cortex: Neuronal encoding of unsigned reward prediction errors driving adjustment in behavior. Journal of Neuroscience, 31(11), 4178-4187.

Hayden, B. Y., Heilbronner, S. R., \& Platt, M. L. (2010). Ambiguity aversion in rhesus macaques. Frontiers in Neuroscience, 4, 166. doi:10.3389/fnins.2010.00166

Hayden, B. Y., Heilbronner, S. R., Nair, A. C., \& Platt, M. L. (2008a). Cognitive influences on risk-seeking by rhesus macaques. Judgment and Decision Making, 3(5), 389-395.

Hayden, B. Y., Nair, A. C., McCoy, A. N., \& Platt, M. L. (2008b). Posterior cingulate cortex mediates outcome-contingent allocation of behavior. Neuron, 60(1), 19-25.

Heilbronner, S. R., \& Hayden, B. Y. (2013). Contextual factors explain risk-seeking preferences in rhesus monkeys. Frontiers in Neuroscience, 7, 7.

Heilbronner, S.R., Hayden, B.Y., \& Platt, M.L. (2009). Neuroeconomis of risk-sensitive decision making. In G. Madden \& W. Bickel (Eds.), Impulsivity: The Behavioral and Neurological Science of Discounting: American Psychological Association. Washington, DC

Heilbronner, S. R., Hayden, B. Y., \& Platt, M. L. (2011). Decision salience signals in posterior cingulate cortex. Frontiers in Neuroscience, 5, 55.

Heilbronner, S. R., Rosati, A. G., Stevens, J. R., Hare, B., \& Hauser, M. D. (2008). A fruit in the hand or two in the bush? Divergent risk preferences in chimpanzees and bonobos. Biology Letters, 4(3), 246-249.

Hertwig, R., Barron, G., Weber, E. U., \& Erev, I. (2004). Decisions from experience and the effect of rare events in risky choice. Psychological Science, 15(8), 534-539.

Hertwig, R., \& Erev, I. (2009). The description-experience gap in risky choice. Trends in Cognitive Science, 13(12), 517-523. doi:10.1016/ j.tics.2009.09.004

Jessup, R. K., Bishara, A. J., \& Busemeyer, J. R. (2008). Feedback produces divergence from prospect theory in descriptive choice. Psychological Science, 19(10), 1015-1022.

Kahneman, D., \& Tversky, A. (1979). Prospect Theory: An analysis of decision under risk. Econometrica, 47, 263-291.

Kidd, C., Piantadosi, S. T., \& Aslin, R. N. (2012). The Goldilocks effect: Human infants allocate attention to visual sequences that are neither too simple nor too complex. PLoS One, 7(5), e36399.

Krupenye, C., Rosati, A. G., \& Hare, B. (2015). Bonobos and chimpanzees exhibit human-like framing effects. Biology Letters, 11(2).

Ludvig, E. A., Madan, C. R., Pisklak, J. M., \& Spetch, M. L. (2014). Reward context determines risky choice in pigeons and humans. Biol Lett, 10(8). doi: 10.1098/rsbl.2014.0451

Ludvig, E. A., Madan, C. R., \& Spetch, M. L. (2015). Priming memories of past wins induces risk seeking. Journal of Experimental Psychology: General, 144(1), 24-29.

Ludvig, E. A., \& Spetch, M. L. (2011). Of black swans and tossed coins: Is the description-experience gap in risky choice limited to rare events? PLoS One, 6(6), e20262. doi:10.1371/journal.pone. 0020262 
Madan, C. R., Ludvig, E. A., \& Spetch, M. L. (2014). Remembering the best and worst of times: Memories for extreme outcomes bias risky decisions. Psychonomic Bulletin and Review, 21(3), 629-636.

McCoy, A. N., \& Platt, M. L. (2005). Risk-sensitive neurons in macaque posterior cingulate cortex. Nature Neuroscience, 8(9), 1220-1227.

Niv, Y., \& Chan, S. (2011). On the value of information and other rewards. Nature Neuroscience, 14(9), 1095-1097.

Pearson, J. M., Hayden, B. Y., \& Platt, M. L. (2010). Explicit information reduces discounting behavior in Monkeys. Frontiers in Psychology, 1, 237.

Pearson, J. M., Hayden, B. Y., Raghavachari, S., \& Platt, M. L. (2009). Neurons in posterior cingulate cortex signal exploratory decisions in a dynamic multi-option choice task. Current Biology, 19(18), 1-6.

Rachlin, H., Raineri, A., \& Cross, D. (1991). Subjective probability and delay. Journal of the Experimental Analysis of Behavior, 55(2), 233 244.

Rosati, A. G., \& Hare, B. (2011). Chimpanzees and bonobos distinguish between risk and ambiguity. Biology Letters, 7(1), 15-18.

Seo, H., \& Lee, D. (2009). Behavioral and neural changes after gains and losses of conditioned reinforcers. Journal of Neuroscience, 29(11), $3627-3641$.

Sharpe, W. (1964). Capital asset prices: A theory of market equilibrium under conditions of risk. Journal of Finance, 19, 425.

Silberberg, A., Murray, P., Christensen, J., \& Asano, T. (1988). Choice in the repeated-gambles experiment. Journal of the Experimental Analysis of Behavior, 50(2), 187-195.
So, N. Y., \& Stuphorn, V. (2010). Supplementary eye field encodes option and action value for saccades with variable reward. Journal of Neurophysiology, 104(5), 2634-2653.

Stauffer, W. R., Lak, A., Bossaerts, P., \& Schultz, W. (2015). Economic choices reveal probability distortion in macaque monkeys. Journal of Neuroscience, 35(7), 3146-3154.

Strait, C. E., Blanchard, T. C., \& Hayden, B. Y. (2014). Reward value comparison via mutual inhibition in ventromedial prefrontal cortex. Neuron, 82(6), 1357-1366.

Strait, C. E., Sleezer, B. J., \& Hayden, B. Y. (2015). Signatures of value comparison in ventral striatum neurons. PLoS Biology, 13(6), e1002173.

Tversky, A., \& Kahneman, D. (1981). The framing of decisions and the psychology of choice. Science, 211, 453-458.

Ungemach, C., Chater, N., \& Stewart, N. (2009). Are probabilities overweighted or underweighted when rare outcomes are experienced (rarely)? Psychological Science, 20(4), 473-479.

Wittmann, B. C., Daw, N. D., Seymour, B., \& Dolan, R. J. (2008). Striatal activity underlies novelty-based choice in humans. Neuron, 58(6), 967-73.

Xu, E. R., \& Kralik, J. D. (2014). Risky business: rhesus monkeys exhibit persistent preferences for risky options. Frontiers in Psychology, 5, 258.

Yechiam, E., \& Busemeyer, J. R. (2006). The effects of foregone payoffs on underweighting small probability events. Journal of Behavioral Decision Making, 19(1), 1-16. 